\title{
THE PERIDYNAMIC EQUATION OF MOTION IN NON-LOCAL ELASTICITY THEORY
}

\author{
Etienne Emmrich $^{1}$ and Olaf Weckner ${ }^{2}$ \\ ${ }^{1}$ Technische Universität Berlin, Institut für Mathematik \\ Straße des 17. Juni 136, 10623 Berlin, Germany \\ e-mail: emmrich@math.tu-berlin.de \\ ${ }^{2}$ Massachusetts Institute of Technology, Department of Mechanical Engineering \\ Cambridge, MA 02139, USA \\ e-mail: olaf@weckner.de
}

Keywords: Long-range interaction, microelastic material, integro-differential equation, energy conservation, well-posedness, quadrature

\begin{abstract}
Non-local theories in solid mechanics that account for effects of long-range interactions -such as the peridynamic modelling introduced by Silling [39] in 2000- have become topical again. The peridynamic theory is based upon a mathematical formulation without any spatial derivative and may cope with discontinuous displacement fields commonly occurring in fracture mechanics.

Beside an overview of the peridynamic modelling and its application, results concerning the mathematical analysis and numerical solution of the governing equation, which is a partial integro-differential equation with second-order time derivative, are presented. Moreover, the question of energy conservation and the comparison of elastic energy in both the peridynamic and the classical theory are discussed.

Concentrating on the description of a linear microelastic material, well-posedness is proved. Based upon the quadrature formula method, a spatial approximation for the numerical solution is suggested. Finally, numerical simulations are presented.
\end{abstract}




\section{INTRODUCTION}

Although non-local theories in continuum mechanics have rather been known since the 1970s from articles by Kröner [29], Edelen, Eringen and co-authors (cf. e.g. [20] and the references cited in [8, 36]), and since 1982 from the books by Kunin [30] and Rogula [38], they have recently become topical again, cf. e.g. [1, 2, 8, 15, 33, 35, 36, 39, 48, 49] and the references cited therein. One of the non-local theories is the so-called peridynamic theory, suggested in Silling [39] in order to describe the formation of discontinuities in the displacement field such as cracks and fractures due to deformations of (micro-) elastic materials. In contrast to the classical local theory but also to other non-local approaches, the peridynamic equation of motion is in integral form and is free of any spatial derivative as it relies on differences of nearby displacements.

Meanwhile, Silling together with Askari, Bobaru, Gerstle, Jiang, and Sau (cf. [11, 12, 21, $22,40,42,43,44])$ have applied the peridynamic modelling to study many examples of solid mechanics such as the Kalthoff-Winkler experiments of the fracture of a steel plate with notches, the cracking growth of a thick plate with an initial inner crack, Hertzian cracks, reinforced concrete beams, the damaging, cracking, and tearing of membranes, the bursting of balloons, and the deformation of fibers and networks of fibers due to long-range forces. In addition to Silling [39], more theoretical considerations regarding the peridynamic modelling, as for instance the dynamics of an infinite bar, the motion of phase boundaries, jump conditions or nonlinear dispersion relations, can also be found in papers by Abeyaratne, Bhattacharya, Dayal, Emmrich, Weckner, and Zimmermann (cf. [17, 19, 47, 50, 51, 52, 53]). The numerical solution of the peridynamic equation of motion has been considered especially in $[19,21,22,43,51,52]$.

In the peridynamic theory, the time evolution of the discplacement $\mathbf{u}$ of a body that occupies the reference volume $\mathcal{V} \subseteq \mathbb{R}^{3}$ in the time interval $(0, T)$ is described by the partial integrodifferential equation (PIDE)

$$
\rho_{0}(\mathbf{x}) \partial_{t}^{2} \mathbf{u}(\mathbf{x}, t)=\int_{\mathcal{H}(\mathbf{x})} \mathbf{f}(\mathbf{x}, \hat{\mathbf{x}}, \mathbf{u}(\mathbf{x}, t), \mathbf{u}(\hat{\mathbf{x}}, t), t) \mathrm{d} \hat{\mathbf{x}}+\mathbf{h}(\mathbf{x}, t),
$$

where $\rho_{0}$ denotes the mass density, $\mathbf{f}$ the pairwise force field that describes the interaction of material particles, and $\mathbf{h}$ collects outer forces. Moreover,

$$
\mathcal{H}(\mathbf{x}):=\{\hat{\mathbf{x}} \in \mathcal{V}:\|\hat{\mathbf{x}}-\mathbf{x}\| \leq \delta\}
$$

is the peridynamic horizon of $\mathrm{x} \in \mathcal{V}$, where $\|\cdot\|$ denotes the Euclidean norm. It is assumed here that the pairwise force function vanishes outside the corresponding horizon although an integration over the whole domain might be the starting point of the model. Moreover, the pairwise force function might be depending on further quantities as e.g. first-order time derivatives. The PIDE (1) is supplemented by initial conditions for $\mathbf{u}(\cdot, 0)$ and $\partial_{t} \mathbf{u}(\cdot, 0)$.

From the mathematical point of view, it is worth mentioning that relatively little is known about integro-differential equations and if so then authors consider integro-differential equations in which the integral is taken over time. Such problems arise when modelling materials with memory or population dynamics with delay (cf. e.g. [14, 31]). Only in [4], PIDEs of the aforementioned type (1) with a linear force function (leading to a so-called Barbashin operator) are considered but with a focus that is outside of our scope. Recently, Emmrich and Weckner [19] have provided a mathematical setting for the treatment of (1) in the linear one-dimensional case that allows to prove existence, uniqueness, and stability results, including the stability with 
respect to the perturbation of the pairwise force function, which justifies its reconstruction from experimental data.

There is also very few known on the numerical solution of (1). With respect to space, one might try to adopt methods known for the approximate solution of integral equations of the second kind (cf. e.g. [6, 13] and the references cited therein). However, there is even less known on the solution of nonlinear integral equations (cf. the survey in Atkinson [7]). The approximation of the integral part leads to an initial value problem for a large coupled system of second-order ordinary differential equations. Beside classical methods such as the Newmark scheme or the leap-frog method, many other methods might be applicable for the time discretisation as much progress has been made in the construction of especially structure-preserving time integrators (cf. e.g. [27, 45, 46] and the references cited therein).

The computational code called Emu (cf. [41]) behind the numerical studies by Silling and co-authors is a quadrature formula method relying on the composite midpoint rule for the approximation of the integral. It uses equidistant cubes of length $\Delta x \approx \delta / 3$. The discretisation in time is an explicit leap-frog method using the central divided difference of second-order with constant time steps. The method falls into the class of meshfree approximations. Moreover, the Emu code uses parallelisation.

For other meshfree methods and similar discrete problems, we refer to the papers in $[25,26]$. Meshfree methods applied to problems with cracking or rupture can also be found e.g. in $[9,16$, 34, 37].

For the peridynamic equation of motion in a one-dimensional unbounded domain, Weckner and Emmrich $[19,51,52]$ have suggested the approximation of the integral, possibly splitted with respect to a priori known discontinuities in the initial values or right-hand side, by means of the Gauß-Hermite and composite midpoint quadrature. For the time discretisation, linear multistep methods with changing order and changing step size have been employed. Moreover, linear finite elements have been considered.

After spatial discretisation, the peridynamic equation of motion resembles the equations arising in molecular dynamics (cf. e.g. [24]). Although peridynamic modelling is meant to describe length scales between those of classical and molecular dynamics, it is a very interesting question whether in the limit $\delta \rightarrow 0$ the classical elasticity theory is recovered and how the spatially discretised peridynamic equation for large $\delta$ is related to molecular dynamics. The discussion of the limit behaviour is also very topical. We refer in particular to the work by Arndt, Blanc, Griebel, Le Bris, Legoll, and Lions (cf. e.g. [5, 10, 32]) on higher-order gradient continuum models, the coupling of atomistic and continuum mechanics by multiscale methods, and the modelling of crystalline structures.

The aim of this paper is to give a short overview of the peridynamic theory. We introduce the peridynamic equation of motion and its corresponding variational formulation and address the question of energy conservation. We then discuss possible choices of the pairwise force function and establish a relation of material paramaters in the peridynamic and in the classical local theory. For the linear case with damping, we furthermore prove well-posedness as well as stability with respect to the data and against a perturbation of the integral kernel. Finally, we suggest the numerical solution via the quadrature formula method based upon a composite Gauß quadrature and present some numerical simulations. 


\section{MODELLING DIFFERENT MATERIAL BEHAVIOUR}

Due to the balance of the linear as well as angular momentum of the massfree bond between $\mathrm{x}$ and $\hat{\mathrm{x}}$,

$$
\begin{gathered}
(\hat{\mathbf{x}}+\mathbf{u}(\hat{\mathbf{x}}, t)) \times \mathbf{f}(\mathbf{x}, \hat{\mathbf{x}}, \mathbf{u}(\mathbf{x}, t), \mathbf{u}(\hat{\mathbf{x}}, t), t) \\
=(\mathbf{x}+\mathbf{u}(\mathbf{x}, t)) \times \mathbf{f}(\mathbf{x}, \hat{\mathbf{x}}, \mathbf{u}(\mathbf{x}, t), \mathbf{u}(\hat{\mathbf{x}}, t), t), \quad \forall \mathbf{x}, \hat{\mathbf{x}} \in \mathcal{V}, t \in(0, T),
\end{gathered}
$$

the pairwise force function $\mathbf{f}$ in (1) has always the direction of the vector pointing from the current position $\mathbf{x}+\mathbf{u}(\mathbf{x}, t)$ to the reference position $\hat{\mathbf{x}}+\mathbf{u}(\hat{\mathbf{x}}, t)$. We can, therefore, write

$$
\mathbf{f}(\mathbf{x}, \hat{\mathbf{x}}, \mathbf{u}, \hat{\mathbf{u}}, t)=f(\mathbf{x}, \hat{\mathbf{x}}, \mathbf{u}, \hat{\mathbf{u}}, t) \mathbf{e}, \quad \mathbf{e}:=\frac{(\hat{\mathbf{x}}+\hat{\mathbf{u}})-(\mathbf{x}+\mathbf{u})}{\|(\hat{\mathbf{x}}+\hat{\mathbf{u}})-(\mathbf{x}+\mathbf{u})\|}
$$

with a scalar function $f$.

If the system is assumed to be invariant against a rigid body motion and if the internal forces are independent of time then

$$
\mathbf{f}(\mathbf{x}, \hat{\mathbf{x}}, \mathbf{u}, \hat{\mathbf{u}}, t)=\mathbf{f}(\mathbf{x}, \hat{\mathbf{x}}, \hat{\mathbf{u}}-\mathbf{u})
$$

with

$$
\mathbf{f}(\hat{\mathbf{x}}, \mathbf{x},-\eta)=-\mathbf{f}(\mathbf{x}, \hat{\mathbf{x}}, \eta)
$$

for all $\mathbf{x}, \hat{\mathbf{x}}, \eta$ because of Newton's law actio et reactio.

If the material is microelastic in the sense of Silling [40] then there exists a pairwise potential $w$ such that

$$
\mathbf{f}(\mathbf{x}, \hat{\mathbf{x}}, \eta)=\nabla_{\eta} w(\mathbf{x}, \hat{\mathbf{x}}, \eta)
$$

The equation of motion (1) then follows from the variational problem

$$
\text { find } \mathbf{u}=\arg \min J(\mathbf{u}) \quad \text { with } \quad J(\mathbf{u}):=\int_{0}^{T} \int_{\mathcal{V}} l(\mathbf{x}, \mathbf{u}(\mathbf{x}, t), t) \mathrm{d} \mathcal{V} \mathrm{d} t,
$$

where $l=e_{\text {kin }}-e_{\mathrm{el}}-e_{\mathrm{ext}}$ is the Lagrangian density and incorporates the density

$$
e_{\text {kin }}=\frac{\rho(\mathbf{x})}{2}\left|\partial_{t} \mathbf{u}(\mathbf{x}, t)\right|^{2}
$$

of the kinetic energy, the elastic energy density

$$
e_{\mathrm{el}}=\frac{1}{2} \int_{\mathcal{H}(\mathbf{x})} w(\mathbf{x}, \hat{\mathbf{x}}, \mathbf{u}(\hat{\mathbf{x}}, t)-\mathbf{u}(\mathbf{x}, t)) \mathrm{d} \hat{\mathcal{V}}
$$

and the density

$$
e_{\mathrm{ext}}=-\mathbf{h}(\mathbf{x}, t) \cdot \mathbf{u}(\mathbf{x}, t)
$$

due to the external force density $\mathbf{h}$ as is shown in [52].

We are now going to present some simple choices for the pairwise force function describing different material behaviour which have been suggested in the literature so far.

A Taylor expansion of $\mathbf{f}$ justifies the linear ansatz

$$
\mathbf{f}(\mathbf{x}, \hat{\mathbf{x}}, \eta)=\mathbf{f}_{\mathbf{0}}(\mathbf{x}, \hat{\mathbf{x}})+\mathbf{C}(\mathbf{x}, \hat{\mathbf{x}}) \cdot \eta
$$


with a stiffness tensor $\mathbf{C}=\mathbf{C}(\mathbf{x}, \hat{\mathbf{x}})$ and $\mathbf{f}_{\mathbf{0}}$ denoting forces in the prestressed reference configuration. For convenience, we only consider $f_{0} \equiv 0$. The linear ansatz (5) is the simplest model we can employ.

In general, the stiffness tensor $\mathbf{C}$ is neither definite nor depending on the length of the difference $\|\hat{\mathbf{x}}-\mathbf{x}\|$, only. It is, however, assumed to be symmetric with respect to its arguments (actio et reactio) as well as symmetric with respect to its tensor structure,

$$
\mathbf{C}(\hat{\mathbf{x}}, \mathbf{x})=\mathbf{C}(\mathbf{x}, \hat{\mathbf{x}}), \quad \mathbf{C}(\mathbf{x}, \hat{\mathbf{x}})^{\top}=\mathbf{C}(\mathbf{x}, \hat{\mathbf{x}}) .
$$

The corresponding micropotential is then given by

$$
w(\mathbf{x}, \hat{\mathbf{x}}, \eta)=\frac{1}{2} \eta \cdot \mathbf{C}(\mathbf{x}, \hat{\mathbf{x}}) \cdot \eta .
$$

In this linear case, the peridynamic equation of motion (1) reads as

$$
\rho(\mathbf{x}) \partial_{t}^{2} \mathbf{u}(\mathbf{x}, t)=\int_{\mathcal{H}(\mathbf{x})} \mathbf{C}(\mathbf{x}, \hat{\mathbf{x}}) \cdot(\mathbf{u}(\hat{\mathbf{x}}, t)-\mathbf{u}(\mathbf{x}, t)) \mathrm{d} \hat{\mathcal{V}}+\mathbf{h}(\mathbf{x}, t),(\mathbf{x}, t) \in \mathcal{V} \times(0, T) .
$$

As we have shown in [52], the total energy is conserved if $\mathbf{h}$ does not depend on $t$,

$$
\frac{\mathrm{d}}{\mathrm{d} t}\left(\mathcal{E}_{\text {kin }}(t)+\mathcal{E}_{\text {el }}(t)+\mathcal{E}_{\text {ext }}(t)\right)=0,
$$

where $\mathcal{E}_{i}(t)=\int_{\mathcal{V}} e_{i}(\mathbf{x}, \mathbf{u}(\mathbf{x}, t), t) \mathrm{d} \mathcal{V}(i \in\{$ kin, el, ext $\})$. In the non-autonomous case, the energy inequality

$$
\begin{gathered}
\mathcal{E}_{\text {kin }}(t)+\mathcal{E}_{\text {el }}(t)+\nu \int_{0}^{t} \mathrm{e}^{\nu(t-s)} \mathcal{E}_{\text {el }}(s) \mathrm{d} s \\
\leq \mathrm{e}^{\nu t}\left(\mathcal{E}_{\text {kin }}(0)+\mathcal{E}_{\text {el }}(0)\right)+\frac{1}{2 \nu} \int_{0}^{t} \int_{\mathcal{V}} \frac{\mathrm{e}^{\nu(t-s)}}{\rho(\mathbf{x})}\|\mathbf{h}(\mathbf{x}, t)\|^{2} \mathrm{~d} \mathcal{V} \mathrm{d} s
\end{gathered}
$$

holds true for all $t \in(0, T)$ and arbitrary $\nu>0$. A similar result can also be found in [39].

If we assume a linear microelastic material then the stiffness tensor can be shown to read as

$$
\mathbf{C}(\mathbf{x}, \hat{\mathbf{x}})=\lambda(\|\hat{\mathbf{x}}-\mathbf{x}\|)(\hat{\mathbf{x}}-\mathbf{x}) \otimes(\hat{\mathbf{x}}-\mathbf{x}),
$$

cf. also [39], where $\lambda$ depends on the specific material model and is e.g. given by

$$
\lambda(\xi)=\frac{c}{\xi^{3}},
$$

cf. also [43]. Here, $c$ denotes a constant that depends again on the material but also on the dimension of the problem. We are going to determine $c$ below.

In order to incorporate nonlinear effects, the proportional microelastic material has been proposed by Silling. Remembering (2) and (3), we suppose that

$$
\mathbf{f}(\mathbf{x}, \hat{\mathbf{x}}, \eta)=f(\mathbf{x}, \hat{\mathbf{x}}, \eta) \mathbf{e}, f(\mathbf{x}, \hat{\mathbf{x}}, \eta)=c s, \quad \hat{\mathbf{x}} \in \mathcal{H}(\mathbf{x}),
$$

where $s$ denotes the bond stretch that is the relative change of the length of a bond (see also Figure 1),

$$
s:=\frac{\|(\hat{\mathbf{x}}+\mathbf{u}(\hat{\mathbf{x}}, t))-(\mathbf{x}+\mathbf{u}(\mathbf{x}, t))\|-\|\hat{\mathbf{x}}-\mathbf{x}\|}{\|\hat{\mathbf{x}}-\mathbf{x}\|} .
$$




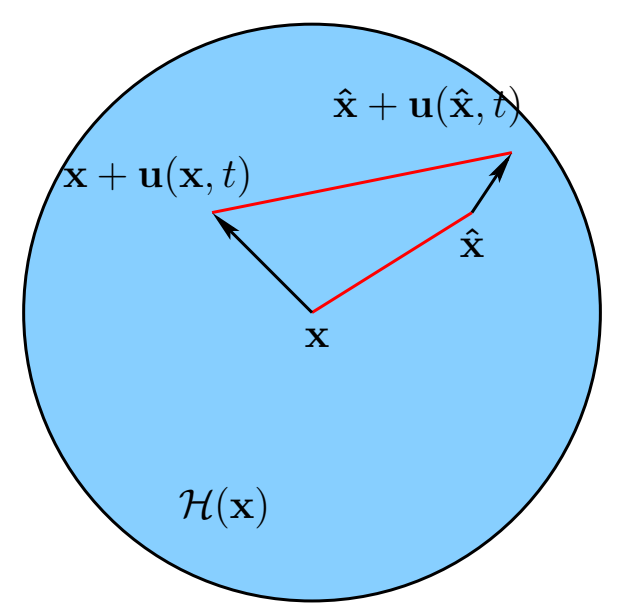

Figure 1: New and old bond and displacements within the peridynamic horizon

Note that

$$
|s| \leq \frac{\|\mathbf{u}(\hat{\mathbf{x}}, t)-\mathbf{u}(\mathbf{x}, t)\|}{\|\hat{\mathbf{x}}-\mathbf{x}\|} .
$$

If necessary we annotate explicitly the arguments of $s$.

The constant of proportionality $c$ depends on the radius of the peridynamic horizon but also on the dimension of the domain $\mathcal{V}$. It can be determined in such a way that the deformation energy of a homogeneous body under isotropic expansion arising from the peridynamic model coincides with the energy known from the classical theory. More precisely, if $\eta:=\mathbf{u}(\hat{\mathbf{x}}, t)-$ $\mathbf{u}(\mathbf{x}, t)=s(\hat{\mathbf{x}}-\mathbf{x})$ (with $s>0)$ then the micropotential $w$ is (up to some additive constant) given by

$$
w(\mathbf{x}, \hat{\mathbf{x}}, \eta)=\frac{c \eta \cdot \eta}{2\|\hat{\mathbf{x}}-\mathbf{x}\|}=\frac{c s^{2}\|\hat{\mathbf{x}}-\mathbf{x}\|}{2} .
$$

This is easily justified since

$$
\nabla_{\eta} w(\mathbf{x}, \hat{\mathbf{x}}, \eta)=c s\|\hat{\mathbf{x}}-\mathbf{x}\| \nabla_{\eta} s=c s \nabla_{\eta}\|\hat{\mathbf{x}}-\mathbf{x}+\eta\|=c s \mathbf{e}=\mathbf{f}(\mathbf{x}, \hat{\mathbf{x}}, \eta) .
$$

From (4), we conclude in the three-dimensional case

$$
e_{\mathrm{el}}=\frac{c s^{2}}{4} \int_{0}^{\delta} \int_{0}^{2 \pi} \int_{0}^{\pi} r^{3} \sin \theta \mathrm{d} \theta \mathrm{d} \phi \mathrm{d} r=\frac{c s^{2} \delta^{4} \pi}{4}
$$

(we assume that the horizon is a full ball). In the classical linear elasticity theory, we have

$$
e_{\mathrm{el}}=\frac{9 k s^{2}}{2}=\frac{3 E s^{2}}{2(1-2 \nu)},
$$

where $k$ denotes the bulk modulus, $\nu$ the Poisson number, and $E$ the Young modulus. As one can also show that $\nu=1 / 4$ in the peridynamic theory, we obtain

$$
c=\frac{12 E}{\pi \delta^{4}}=\frac{18 k}{\pi \delta^{4}}
$$

In the two-dimensional case, we find analogously

$$
c=\frac{72 k}{5 \pi \delta^{3}}
$$


and in the one-dimensional case

$$
c=\frac{18 k}{5 \delta^{2}}
$$

A linearisation of the pairwise force function (9) of the proportional microelastic material gives exactly the linear model (5) with the stiffness tensor $\mathbf{C}$ given by (7) and (8) and the constant of proportionality $c$ (depending on the dimension of $\mathcal{V}$ ) as above.

A next step in the modelling is to incorporate breaking bonds in order to describe damage and fracture. The simplest approach is the so-called prototype microelastic brittle material (cf. $[42,43,44])$ that relies upon the idea of the proportional microelastic material but allows bond breakage (see also Figure 2),

$f(\mathbf{x}, \hat{\mathbf{x}}, \mathbf{u}(\mathbf{x}, t), \mathbf{u}(\hat{\mathbf{x}}, t), t)= \begin{cases}c s(\mathbf{x}, \hat{\mathbf{x}}, \mathbf{u}(\mathbf{x}, t), \mathbf{u}(\hat{\mathbf{x}}, t)) & \text { if } \hat{\mathbf{x}} \in \mathcal{H}(\mathbf{x}) \text { and } \\ & s(\mathbf{x}, \hat{\mathbf{x}}, \mathbf{u}(\mathbf{x}, \tau), \mathbf{u}(\hat{\mathbf{x}}, \tau)) \leq s_{0} \forall \tau \leq t \\ 0 & \text { else }\end{cases}$

Here $s=s(\mathbf{x}, \hat{\mathbf{x}}, \mathbf{u}(\mathbf{x}, t), \mathbf{u}(\hat{\mathbf{x}}, t))$ again denotes the bond stretch but note that it implicitly depends on time. Moreover, $s_{0}$ is some given critical bond stretch that might be determined from experimental data. In [43],

$$
s_{0}=\sqrt{\frac{5 G_{0}}{9 k \delta}}
$$

is suggested for the three-dimensional case with the work $G_{0}$ required to break all the bonds per unit fracture area (energy release rate). If a bond is broken then it remains broken for all future time.

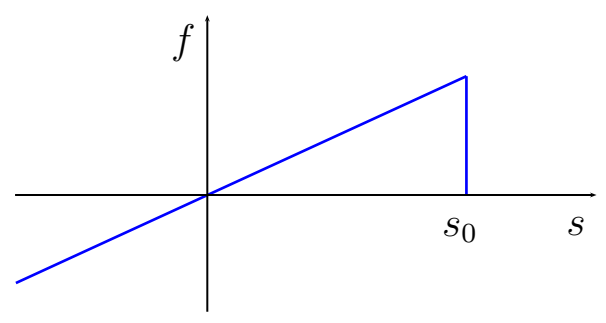

Figure 2: Prototype microelastic brittle material

Further material models such as the proportional microplastic one have also been suggested in the literature.

\section{MATHEMATICAL ANALYSIS IN THE LINEAR CASE}

Recently, we have proven well-posedness in the linear, one-dimensional, unbounded case (cf. [19]). In the following, we employ the same technique of proof to establish existence, uniqueness, and stability results as well as a priori estimates in the linear case with damping for a bounded domain of arbitrary dimension.

More precisely, we consider for $(\mathbf{x}, t) \in \mathcal{V} \times(0, T)$ the initial-value problem for the more general PIDE

$$
\partial_{t}^{2} \mathbf{u}(\mathbf{x}, t)+r \partial_{t} \mathbf{u}(\mathbf{x}, t)=\int_{\mathcal{V}} \mathbf{K}(\mathbf{x}, \hat{\mathbf{x}}) \cdot \mathbf{u}(\hat{\mathbf{x}}, t) \mathrm{d} \hat{\mathcal{V}}+\mathbf{K}_{\mathbf{0}}(\mathbf{x}) \cdot \mathbf{u}(\mathbf{x}, t)+\mathbf{b}(\mathbf{x}, t)
$$


with a bounded domain $\mathcal{V} \subset \mathbb{R}^{d}(d \in\{1,2,3\})$, the time interval $(0, T)$, and a damping parameter $r \geq 0$. Here, $\mathbf{K}: \overline{\mathcal{V}} \times \overline{\mathcal{V}} \rightarrow \mathbb{R}^{d \times d}, \mathbf{K}_{\mathbf{0}}: \overline{\mathcal{V}} \rightarrow \mathbb{R}^{d \times d}$, and $\mathbf{b}: \overline{\mathcal{V}} \times[0, T] \rightarrow \mathbb{R}^{d}$ are given matrix- and vector-valued functions, respectively. Equation (11) coincides with the linear peridynamic equation of motion (6) if $r=0$ and

$$
\mathbf{K}(\mathbf{x}, \hat{\mathbf{x}})=\left\{\begin{array}{ll}
\frac{\mathbf{C}(\mathbf{x}, \hat{\mathbf{x}})}{\rho(\mathbf{x})} & \text { if } \hat{\mathbf{x}} \in \mathcal{H}(\mathbf{x}) \\
0 & \text { else }
\end{array}, \mathbf{K}_{\mathbf{0}}(\mathbf{x})=-\int_{\mathcal{V}} \mathbf{K}(\mathbf{x}, \hat{\mathbf{x}}) \mathrm{d} \hat{\mathcal{V}}, \mathbf{b}(\mathbf{x}, t)=\frac{\mathbf{h}(\mathbf{x}, t)}{\rho(\mathbf{x})}\right.
$$

but it also includes the more general case where $\mathbf{K}$ does not vanish outside the peridynamic horizon. Whether the assumptions imposed on $\mathbf{K}$ and $\mathbf{K}_{\mathbf{0}}$ in the following are fulfilled for a stiffness tensor $\mathbf{C}$ given by (7) and (8) will be discussed at the end of this section.

Let $X$ be a suitable Banach space of functions depending on $\mathbf{x} \in \overline{\mathcal{V}}$ mapping into $\mathbb{R}^{d}$ that will be specified below. With the $\mathbb{R}^{d}$-valued functions $\mathbf{u}=\mathbf{u}(\mathbf{x}, t): \overline{\mathcal{V}} \times[0, T] \rightarrow \mathbb{R}^{d}$ and $\mathbf{b}=\mathbf{b}(\mathbf{x}, t): \overline{\mathcal{V}} \times[0, T] \rightarrow \mathbb{R}^{d}$, we can associate the $X$-valued abstract functions $u=u(t)$ : $[0, T] \rightarrow X$ and $b=b(t):[0, T] \rightarrow X$. Equation (11) can then be rewritten as the second-order operator-differential equation in $X$

$$
\ddot{u}(t)+r \dot{u}(t)=A u(t)+b(t), t \in(0, T),
$$

or as the first-order operator-differential equation in $X \times X$

$$
\frac{\mathrm{d}}{\mathrm{d} t}\left(\begin{array}{c}
u(t) \\
\dot{u}(t)
\end{array}\right)=\mathcal{A}(t)\left(\begin{array}{c}
u(t) \\
\dot{u}(t)
\end{array}\right)+\left(\begin{array}{c}
0 \\
b(t)
\end{array}\right), t \in(0, T), \quad \mathcal{A}:=\left(\begin{array}{cc}
0 & \text { id } \\
A & -r \mathrm{id}
\end{array}\right) .
$$

Here, id denotes the identity in $X$ and $A=K+K_{0}$ is the sum of the integral operator $K$ and the multiplication operator $K_{0}$, which are given by

$$
(K \mathbf{v})(\mathbf{x}):=\int_{\mathcal{V}} \mathbf{K}(\mathbf{x}, \hat{\mathbf{x}}) \cdot \mathbf{v}(\hat{\mathbf{x}}) \mathrm{d} \hat{\mathcal{V}},\left(K_{0} \mathbf{v}\right)(\mathbf{x}):=\mathbf{K}_{\mathbf{0}}(\mathbf{x}) \cdot \mathbf{v}(\mathbf{x}), \mathbf{v} \in X .
$$

Moreover, a dot means the derivative of an abstract function with respect to time $t$.

In order to justify (12) and (13), we show that $A$ maps $X$ into $X$. This, however, depends on the choice of the space $X$. In the following, we consider the case $X=L^{p}(\mathcal{V})^{d}(p \in[1, \infty])$, the space of $\mathbb{R}^{d}$-valued functions on $\mathcal{V}$ whose components are Lebesgue-measurable and for which the $p$-th power of the absolute value of the components is integrable if $1 \leq p<\infty$ and the absolute value of the components is essentially bounded if $p=\infty$, respectively, equipped with the norm

$$
\|\mathbf{v}\|_{L^{p}(\mathcal{V})^{d}}:= \begin{cases}\left(\int_{\mathcal{V}}\|\mathbf{v}(\mathbf{x})\|^{p} \mathrm{~d} \mathcal{V}\right)^{1 / p} & \text { if } 1 \leq p<\infty, \\ \operatorname{ess} \sup _{\mathbf{x} \in \mathcal{V}}\|\mathbf{v}(\mathbf{x})\| & \text { if } p=\infty .\end{cases}
$$

We make also use of other $L^{p}$-spaces whose definition is analogous.

Here and in the following, $\|\cdot\|$ denotes again the Euclidean length but also the corresponding spectral matrix norm. Note that by definition

$$
\|\mathbf{A} \cdot \mathbf{v}\| \leq\|\mathbf{A}\|\|\mathbf{v}\|
$$

holds for all matrices $\mathbf{A} \in \mathbb{R}^{d \times d}$ and vectors $\mathbf{v} \in \mathbb{R}^{d}$. 
Proposition 3.1. Let $\mathbf{K}_{\mathbf{0}} \in L^{\infty}(\mathcal{V})^{d \times d}$, let $\mathbf{K}$ be Lebesgue-measurable on $\mathcal{V} \times \mathcal{V}$, and assume that for some $p \in[1, \infty]$ (with the conjugated exponent $q, 1 / p+1 / q=1$ )

$$
\kappa_{0}:=\operatorname{ess} \sup _{\mathbf{x} \in \mathcal{V}}\left\|\mathbf{K}_{\mathbf{0}}(\mathbf{x})\right\|<\infty, \quad \kappa<\infty
$$

where

$$
\kappa:= \begin{cases}\int_{\mathcal{V}} \operatorname{ess} \sup _{\hat{\mathbf{x}} \in \mathcal{V}}\|\mathbf{K}(\mathbf{x}, \hat{\mathbf{x}})\| \mathrm{d} \mathcal{V} & \text { if } p=1, \\ \left(\int_{\mathcal{V}}\left(\int_{\mathcal{V}}\|\mathbf{K}(\mathbf{x}, \hat{\mathbf{x}})\|^{q} \mathrm{~d} \hat{\mathcal{V}}\right)^{p / q} \mathrm{~d} \mathcal{V}\right)^{1 / p} & \text { if } 1<p<\infty, \\ \operatorname{ess} \sup _{\mathbf{x} \in \mathcal{V}} \int_{\mathcal{V}}\|\mathbf{K}(\mathbf{x}, \hat{\mathbf{x}})\| \mathrm{d} \hat{\mathcal{V}} & \text { if } p=\infty .\end{cases}
$$

Then the operators $A, K$, and $K_{0}$ are linear bounded mappings in $L^{p}(\mathcal{V})^{d}$, and the operator $\mathcal{A}$ is a linear bounded mapping in $L^{p}(\mathcal{V})^{d} \times L^{p}(\mathcal{V})^{d}$.

Proof. Let $\mathbf{v} \in L^{p}(\mathcal{V})^{d}(p \in(1, \infty))$. It is clear that $(\mathbf{x}, \hat{\mathbf{x}}) \mapsto \mathbf{K}(\mathbf{x}, \hat{\mathbf{x}}) \cdot \mathbf{v}(\mathbf{x})$ as well as $\mathbf{x} \mapsto \mathbf{K}_{\mathbf{0}}(\mathbf{x}) \cdot \mathbf{v}(\mathbf{x})$ are Lebesgue-measurable functions. With Hölder's inequality, it holds

$$
\begin{gathered}
\|K \mathbf{v}\|_{L^{p}(\mathcal{V})^{d}}=\left(\int_{\mathcal{V}}\left\|\int_{\mathcal{V}} \mathbf{K}(\mathbf{x}, \hat{\mathbf{x}}) \cdot \mathbf{v}(\hat{\mathbf{x}}) \mathrm{d} \hat{\mathcal{V}}\right\|^{p} \mathrm{~d} \mathcal{V}\right)^{1 / p} \\
\leq\left(\int_{\mathcal{V}}\left(\int_{\mathcal{V}}\|\mathbf{K}(\mathbf{x}, \hat{\mathbf{x}}) \cdot \mathbf{v}(\hat{\mathbf{x}})\| \mathrm{d} \hat{\mathcal{V}}\right)^{p} \mathrm{~d} \mathcal{V}\right)^{1 / p} \leq\left(\int_{\mathcal{V}}\left(\int_{\mathcal{V}}\|\mathbf{K}(\mathbf{x}, \hat{\mathbf{x}})\|\|\mathbf{v}(\hat{\mathbf{x}})\| \mathrm{d} \hat{\mathcal{V}}\right)^{p} \mathrm{~d} \mathcal{V}\right)^{1 / p} \\
\leq\left(\int_{\mathcal{V}}\left(\left(\int_{\mathcal{V}}\|\mathbf{K}(\mathbf{x}, \hat{\mathbf{x}})\|^{q} \mathrm{~d} \hat{\mathcal{V}}\right)^{p / q} \int_{\mathcal{V}}\|\mathbf{v}(\hat{\mathbf{x}})\|^{p} \mathrm{~d} \hat{\mathcal{V}}\right) \mathrm{d} \mathcal{V}\right)^{1 / p}=\kappa\|\mathbf{v}\|_{L^{p}(\mathcal{V})^{d}}
\end{gathered}
$$

Since $K$ is obviously linear, this not only shows that $K$ maps $L^{p}(\mathcal{V})^{d}$ into itself but also that $K$ is bounded. The cases $p=1$ and $p=\infty$ are analogous although somewhat simpler.

Similarly, we have

$$
\left\|K_{0} \mathbf{v}\right\|_{L^{p}(\mathcal{V})^{d}}=\left(\int_{\mathcal{V}}\left\|\mathbf{K}_{\mathbf{0}}(\mathbf{x}) \cdot \mathbf{v}(\mathbf{x})\right\|^{p} \mathrm{~d} \mathcal{V}\right)^{1 / p} \leq \kappa_{0}\|\mathbf{v}\|_{L^{p}(\mathcal{V})^{d}},
$$

which proves the assertion for $K_{0}$.

It immediately follows that $A: L^{p}(\mathcal{V})^{d} \rightarrow L^{p}(\mathcal{V})^{d}$ is linear and bounded with

$$
\|A \mathbf{v}\|_{L^{p}(\mathcal{V})^{d}} \leq \alpha\|\mathbf{v}\|_{L^{p}(\mathcal{V})^{d}}, \alpha:=\kappa+\kappa_{0} .
$$

If we equip the function space $L^{p}(\mathcal{V})^{d} \times L^{p}(\mathcal{V})^{d}$ with the standard norm

$$
\left\|(\mathbf{v}, \mathbf{w})^{\top}\right\|_{L^{p}(\mathcal{V})^{d} \times L^{p}(\mathcal{V})^{d}}:=\|\mathbf{v}\|_{L^{p}(\mathcal{V})^{d}}+\|\mathbf{w}\|_{L^{p}(\mathcal{V})^{d}},
$$

we find

$$
\begin{gathered}
\left\|\mathcal{A}(\mathbf{v}, \mathbf{w})^{\top}\right\|_{L^{p}(\mathcal{V})^{d} \times L^{p}(\mathcal{V})^{d}}=\left\|(\mathbf{w}, A \mathbf{v}-r \mathbf{w})^{\top}\right\|_{L^{p}(\mathcal{V})^{d} \times L^{p}(\mathcal{V})^{d}} \\
\leq \alpha\|\mathbf{v}\|_{L^{p}(\mathcal{V})^{d}}+(1+r)\|\mathbf{w}\|_{L^{p}(\mathcal{V})^{d}} \leq \max (\alpha, 1+r)\left\|(\mathbf{v}, \mathbf{w})^{\top}\right\|_{L^{p}(\mathcal{V})^{d} \times L^{p}(\mathcal{V})^{d}}
\end{gathered}
$$

that finally shows the boundedness of the linear operator $\mathcal{A}: L^{p}(\mathcal{V})^{d} \times L^{p}(\mathcal{V})^{d} \rightarrow L^{p}(\mathcal{V})^{d} \times$ $L^{p}(\mathcal{V})^{d}$. 
By standard arguments (cf. e.g. Amann [3, pp. 151ff.] or Emmrich [18, pp. 173ff.]), it follows that $\mathcal{A}$ generates the uniformly continuous one-parameter group $\{\exp (t \mathcal{A})\}_{t \in \mathbb{R}}$ in $L^{p}(\mathcal{V})^{d} \times$ $L^{p}(\mathcal{V})^{d}$ with

$$
\exp (t \mathcal{A}):=\sum_{n=0}^{\infty} \frac{t^{n}}{n !}\left(\begin{array}{cc}
0 & \mathrm{id} \\
A & -r \mathrm{id}
\end{array}\right)^{n}
$$

and the solution to (12) and (13), respectively, is given by Duhamel's principle. Note that the representation of $\exp (t \mathcal{A})$ simplifies if $r=0$ :

$$
\exp (t \mathcal{A}) \stackrel{r=0}{=}\left(\begin{array}{cc}
\sum_{n=0}^{\infty} \frac{t^{2 n} A^{n}}{(2 n) !} & \sum_{n=0}^{\infty} \frac{t^{2 n+1} A^{n}}{(2 n+1) !} \\
\sum_{n=0}^{\infty} \frac{t^{2 n+1} A^{n+1}}{(2 n+1) !} & \sum_{n=0}^{\infty} \frac{t^{2 n} A^{n}}{(2 n) !}
\end{array}\right) .
$$

We thus come up with the following theorem:

Theorem 3.1. Under the assumptions of Proposition 3.1, there is for given $\mathbf{u}_{\mathbf{0}}, \mathbf{v}_{\mathbf{0}} \in L^{p}(\mathcal{V})^{d}$, $b \in L^{1}\left(0, T ; L^{p}(\mathcal{V})^{d}\right)$ a unique mild solution $u \in \mathcal{C}^{1}\left([0, T] ; L^{p}(\mathcal{V})^{d}\right)$ to the initial-value problem for (12) and (13), respectively, with $u(0)=\mathbf{u}_{0}$ and $\dot{u}(0)=\mathbf{v}_{0}$, which is given by

$$
\left(\begin{array}{c}
u(t) \\
\dot{u}(t)
\end{array}\right)=\exp (t \mathcal{A})\left(\begin{array}{c}
\mathbf{u}_{\mathbf{0}} \\
\mathbf{v}_{\mathbf{0}}
\end{array}\right)+\int_{0}^{t} \exp ((t-s) \mathcal{A})\left(\begin{array}{c}
0 \\
b(s)
\end{array}\right) \mathrm{d} s, t \in(0, T) .
$$

If $r=0$ then for all $t \in(0, T)$

$$
u(t)=\cosh (t \sqrt{A}) \mathbf{u}_{\mathbf{0}}+\sqrt{A}^{-1} \sinh (t \sqrt{A}) \mathbf{v}_{\mathbf{0}}+\sqrt{A}^{-1} \int_{0}^{t} \sinh ((t-s) \sqrt{A}) b(s) \mathrm{d} s,
$$

with the suggestive notation

$$
\cosh (t \sqrt{A}):=\sum_{n=0}^{\infty} \frac{t^{2 n} A^{n}}{(2 n) !}, \sqrt{A}-1 \sinh (t \sqrt{A}):=\sum_{n=0}^{\infty} \frac{t^{2 n+1} A^{n}}{(2 n+1) !} .
$$

If $b \in \mathcal{C}\left([0, T] ; L^{p}(\mathcal{V})^{d}\right)$ then $u \in \mathcal{C}^{2}\left([0, T] ; L^{p}(\mathcal{V})^{d}\right)$.

Here, $L^{1}\left(0, T ; L^{p}(\mathcal{V})^{d}\right)$ denotes the Banach space of Bochner-integrable abstract functions with values in $L^{p}(\mathcal{V})^{d}$ (roughly spoken the space of functions that are integrable with respect to time and square-integrable with respect to space) and $\mathcal{C}^{m}\left([0, T] ; L^{p}(\mathcal{V})^{d}\right)(m \in \mathbb{N})$ is the Banach space of $m$-times continuously differentiable abstract functions with values in $L^{p}(\mathcal{V})^{d}$ (cf. e.g. [18] for more details).

Also regularity results may be obtained which then show the existence and uniqueness of a classical solution to (11).

Regarding the representation (15) as well as a priori estimates in the case of damping, it might be useful to transform the damped system into a system without damping: Let

$$
\tilde{u}(t):=\mathrm{e}^{r t / 2} u(t), \tilde{b}(t):=\mathrm{e}^{r t / 2} b(t), \tilde{A}:=A+\frac{r^{2}}{4} \mathrm{id} .
$$

A short calculation shows that (12) then is equivalent to

$$
\ddot{\tilde{u}}(t)=\tilde{A} \tilde{u}(t)+\tilde{b}(t), t \in(0, T) .
$$


Hence, the representation (16) applied to $\tilde{u}$ yields

$$
\begin{aligned}
& u(t)=\mathrm{e}^{-r t / 2} \cosh \left(t \sqrt{A+\frac{r^{2}}{4} \mathrm{id}}\right) \mathbf{u}_{\mathbf{0}}+\mathrm{e}^{-r t / 2} \sqrt{A+\frac{r^{2}}{4} \mathrm{id}}-1 \sinh \left(t \sqrt{A+\frac{r^{2}}{4} \mathrm{id}}\right) \mathbf{v}_{\mathbf{0}} \\
& +{\sqrt{A+\frac{r^{2}}{4} \mathrm{id}}}^{-1} \int_{0}^{t} \mathrm{e}^{-r(t-s) / 2} \sinh \left((t-s) \sqrt{A+\frac{r^{2}}{4} \mathrm{id}}\right) b(s) \mathrm{d} s, t \in(0, T) .
\end{aligned}
$$

We are now going to establish an a priori estimate for the solution. Since the problem under consideration is linear, this estimate also proves stability with respect to the the initial data and right-hand side.

Theorem 3.2. Under the assumptions of Proposition 3.1 and Theorem 3.1, the following a priori estimate holds true for all $t \in(0, T)$ :

$$
\begin{aligned}
\|u(t)\|_{L^{p}(\mathcal{V})^{d}} & \leq \mathrm{e}^{-r t / 2} \cosh \left(t \sqrt{\alpha+\frac{r^{2}}{4}}\right)\left\|\mathbf{u}_{\mathbf{0}}\right\|_{L^{p}(\mathcal{V})^{d}} \\
& +\mathrm{e}^{-r t / 2} \sqrt{\alpha+\frac{r^{2}}{4}}-1 \sinh \left(t \sqrt{\alpha+\frac{r^{2}}{4}}\right)\left\|\mathbf{v}_{\mathbf{0}}\right\|_{L^{p}(\mathcal{V})^{d}} \\
& +\sqrt{\alpha+\frac{r^{2}}{4}} \int_{0}^{t} \mathrm{e}^{-r(t-s) / 2} \sinh \left((t-s) \sqrt{\alpha+\frac{r^{2}}{4}}\right)\|b(s)\|_{L^{p}(\mathcal{V})^{d}} \mathrm{~d} s .
\end{aligned}
$$

Proof. The estimate is a direct consequence of the representation (18) together with (14).

The a priori estimate above shows indeed the damping effect of the term $r \partial_{t} \mathbf{u}$.

As we have described in [19], it might be possible to determine the integral kernel from experimental data. It is, therefore, the question whether the solution is stable against perturbations of $\mathbf{K}$ and $\mathbf{K}_{\mathbf{0}}$. This can be answered in the affirmative by the following theorem.

Theorem 3.3. Let $b \in L^{1}\left(0, T ; L^{p}(\mathcal{V})^{d}\right)$ and let, for arbitrary $\varepsilon>0, \mathbf{K}, \mathbf{K}_{\mathbf{0}}, \mathbf{K}_{\varepsilon}, \mathbf{K}_{\mathbf{0} \varepsilon}$, which are assumed to fulfill the assumptions of Proposition 3.1, such that

$$
\Delta+\operatorname{ess} \sup _{\mathbf{x} \in \mathcal{V}}\left\|\mathbf{K}_{\mathbf{0}}(\mathbf{x})-\mathbf{K}_{\mathbf{0} \varepsilon}(\mathbf{x})\right\|<\varepsilon
$$

where

$$
\Delta:= \begin{cases}\int_{\mathcal{V}} \operatorname{ess} \sup _{\hat{\mathbf{x}} \in \mathcal{V}}\left\|\mathbf{K}(\mathbf{x}, \hat{\mathbf{x}})-\mathbf{K}_{\varepsilon}(\mathbf{x}, \hat{\mathbf{x}})\right\| \mathrm{d} \mathcal{V} & \text { if } p=1 \\ \left(\int_{\mathcal{V}}\left(\int_{\mathcal{V}}\left\|\mathbf{K}(\mathbf{x}, \hat{\mathbf{x}})-\mathbf{K}_{\varepsilon}(\mathbf{x}, \hat{\mathbf{x}})\right\|^{q} \mathrm{~d} \hat{\mathcal{V}}\right)^{p / q} \mathrm{~d} \mathcal{V}\right)^{1 / p} & \text { if } 1<p<\infty \\ \operatorname{ess} \sup _{\mathbf{x} \in \mathcal{V}} \int_{\mathcal{V}}\left\|\mathbf{K}(\mathbf{x}, \hat{\mathbf{x}})-\mathbf{K}_{\varepsilon}(\mathbf{x}, \hat{\mathbf{x}})\right\| \mathrm{d} \hat{\mathcal{V}} & \text { if } p=\infty\end{cases}
$$


The corresponding mild solutions $u, u_{\varepsilon}$ to equation (12) with initial data $\mathbf{u}_{\mathbf{0}}, \mathbf{v}_{\mathbf{0}} \in L^{p}(\mathcal{V})^{d}$ then satisfy for all $t \in(0, T)$ the estimate

$$
\begin{gathered}
\left\|u(t)-u_{\varepsilon}(t)\right\|_{L^{p}(\mathcal{V})^{d}} \leq \varepsilon{\sqrt{\alpha+\frac{r^{2}}{4}+\varepsilon}}^{-1} \times \\
\times \int_{0}^{t} \mathrm{e}^{-r(t-s) / 2} \sinh \left((t-s) \sqrt{\alpha+\frac{r^{2}}{4}+\varepsilon}\right)\|u(s)\|_{L^{p}(\mathcal{V})^{d}} \mathrm{~d} s .
\end{gathered}
$$

Proof. The proof follows the same lines as in [19, Thm. 3.2] if one replaces the solution space $L^{\infty}(\mathbb{R})$ used there by $L^{p}(\mathcal{V})^{d}$. Moreover, the damping term has to be incorporated here.

Let us consider now the linear peridyamic equation (6) with constant density $\rho$ and with a stiffness tensor $\mathbf{C}$ given by (7) and, in particular, (8). Since

$$
\|\mathbf{C}(\mathbf{x}, \hat{\mathbf{x}})\|=\frac{c}{\|\hat{\mathbf{x}}-\mathbf{x}\|^{3}}\|(\hat{\mathbf{x}}-\mathbf{x}) \otimes(\hat{\mathbf{x}}-\mathbf{x})\|=\frac{c}{\|\hat{\mathbf{x}}-\mathbf{x}\|},
$$

the corresponding integral kernel $\mathbf{K}$ is weakly singular if $d \in\{2,3\}$ but strongly singular if $d=1$.

Unfortunately, we cannot apply the mathematical setting above in the one-dimensional case since then $\mathbf{K}(\mathbf{x}, \hat{\mathbf{x}})$ is not integrable and the assumptions of Proposition 3.1 are not fulfilled. Note that we have considered different integral kernels in [19] satisfying the assumptions of Proposition 3.1 with $p=\infty$ and $q=1$. These integral kernels describe, however, a different material behaviour.

In the two-dimensional case, we can apply the above setting with any $p>2$ as then $q<2$ and (without loss of generality for a horizon $\mathcal{H}(\mathbf{x})$ that is a full ball)

$$
\int_{\mathcal{V}}\|\mathbf{K}(\mathbf{x}, \hat{\mathbf{x}})\|^{q} \mathrm{~d} \hat{\mathcal{V}}=\left(\frac{c}{\rho}\right)^{q} \int_{\mathcal{H}(\mathbf{x})} \frac{1}{\|\hat{\mathbf{x}}-\mathbf{x}\|^{q}} \mathrm{~d} \hat{\mathcal{V}}=\left(\frac{c}{\rho}\right)^{q} \int_{0}^{\delta} \int_{0}^{2 \pi} r^{1-q} \mathrm{~d} r \mathrm{~d} \phi<\infty
$$

as well as

$$
\left\|\mathbf{K}_{\mathbf{0}}(\mathbf{x})\right\| \leq \frac{c}{\rho} \int_{\mathcal{H}(\mathbf{x})} \frac{1}{\|\hat{\mathbf{x}}-\mathbf{x}\|} \mathrm{d} \hat{\mathcal{V}}=\frac{2 \pi c \delta}{\rho} .
$$

Analogously, the mathematical setting above works fine for the three-dimensional case with any $p>3 / 2$ as then $q<3$ (and $r^{2-q}$ is integrable). In particular, we then have $L^{2}$-solvability (i.e. $p=q=2$ ) of the initial-value problem for (6) with (7) and (8).

\section{NUMERICAL SOLUTION AND SIMULATIONS}

For the numerical solution of (1), we suggest a quadrature formula method. Let us suppose we are given $N \in \mathbb{N}$ quadrature points $\mathbf{x}_{i} \in \mathcal{V}(i=1,2, \ldots, N)$. Any quadrature then reads as

$$
\int_{\mathcal{V}} \Phi(\hat{\mathbf{x}}) \mathrm{d} \hat{\mathcal{V}} \approx \sum_{j=1}^{N} \sigma_{j} \Phi\left(\mathbf{x}_{j}\right)
$$

with suitable quadrature weights $\sigma_{j}$. For $i \in\{1,2, \ldots, N\}$, let

$$
I(i):=\left\{j \in\{1,2, \ldots, N\}: \mathbf{x}_{j} \in \mathcal{H}\left(\mathbf{x}_{i}\right)\right\}
$$


be the set of indices describing the quadrature points interacting with $\mathbf{x}_{i}$. It follows

$$
\int_{\mathcal{H}\left(\mathbf{x}_{i}\right)} \Phi(\hat{\mathbf{x}}) \mathrm{d} \hat{\mathcal{V}} \approx \sum_{j \in I(i)} \sigma_{j} \Phi\left(\mathbf{x}_{j}\right)
$$

In the quadrature formula method, we now solve the original equation (1) only at the quadrature points which leads to a large coupled system of nonlinear ordinary differential equations. To be precise, we look for approximations $\mathbf{u}_{i}(t) \approx \mathbf{u}\left(\mathbf{x}_{i}, t\right)(i=1,2, \ldots, N)$ satisfying

$$
\rho\left(\mathbf{x}_{i}\right) \ddot{\mathbf{u}}_{i}(t)=\sum_{j \in I(i)} \sigma_{j} \mathbf{f}\left(\mathbf{x}_{i}, \mathbf{x}_{j}, \mathbf{u}_{i}(t), \mathbf{u}_{j}(t), t\right)+\mathbf{h}\left(\mathbf{x}_{i}, t\right), t \in(0, T) .
$$

In [52], we have proven the conservation of the discrete total energy in the autonomous, linear, one-dimensional case for any quadrature formula and Galerkin-type (e.g. finite element) method.

Possible choices for the quadrature are e.g. the composite midpoint rule or a composite Gauß quadrature on tetrahedrons or cuboids. For simplicity, we confine ourselves to the twodimensional case and the composite midpoint and 4-point-Gauß rule relying on rectangles. Both quadratures can take place at the same time. The midpoint rule is exact for bilinear polynomials. The 4-point-Gauß quadrature is a cross-product rule relying on the one-dimensional quadrature

$$
\int_{-1}^{1} \Phi(x) \mathrm{d} x \approx \Phi\left(-\frac{\sqrt{3}}{3}\right)+\Phi\left(\frac{\sqrt{3}}{3}\right) .
$$

It is exact for bicubic polynomials. For the quadrature on an arbitrary rectangle, we only have to map affine-linearly the four Gauß points $( \pm \sqrt{3} / 3, \pm \sqrt{3} / 3)$.

We assume that the rectangular domain $\mathcal{V}$ is partitioned into the (non-overlapping) rectangles $T_{l}(l=1,2, \ldots, M ; N \leq M)$ with area $\left|T_{l}\right|$. On one rectangle, we have the quadrature

$$
\int_{T_{l}} \Phi(\hat{\mathbf{x}}) \mathrm{d} \hat{\mathcal{V}} \approx \sum_{\mathbf{x}_{k} \in T_{l}} \sigma_{k} \Phi\left(\mathbf{x}_{k}\right)
$$

with

$$
\sigma_{k}:= \begin{cases}\left|T_{l}\right| & \text { if the midpoint rule is used on } T_{l}, \\ \frac{\left|T_{l}\right|}{4} & \text { if the 4-point-Gauß rule is used on } T_{l} .\end{cases}
$$

Summing up over all quadrature points lying in the peridynamic horizon gives the composite quadrature. A possible covering of the peridynamic horizon together with the Gauß points is shown in Figure 3.

As an example, we have solved numerically the peridynamic equation of motion with the proportional microelastic material model (without outer forces and without damping) based upon the composite 4 -point-Gauß quadrature on $24 \times 8$ equidistantly distributed rectangles. We have considered a steel plate with density $\rho \equiv 8000 \mathrm{kgm}^{-3}$, Young modulus $E=210 \cdot 10^{9} \mathrm{~Pa}$, and Poisson number $\nu=1 / 4$ of dimension $1 \mathrm{~m} \times 0.3 \mathrm{~m}$. As the constant of proportionality, (10) has been employed (with $k=E / 3(1-2 \nu)$ ). The initial displacement is zero whereas the $y$-component of the initial velocity vanishes and the $x$-component of the initial velocity is given by $10(x-0.5) \mathrm{ms}^{-1}$. This models an initial tension in $x$-direction. Figures 4 and 5 show the results with a total of 768 quadrature points. The peridynamic horizon is $\delta=0.1$. 


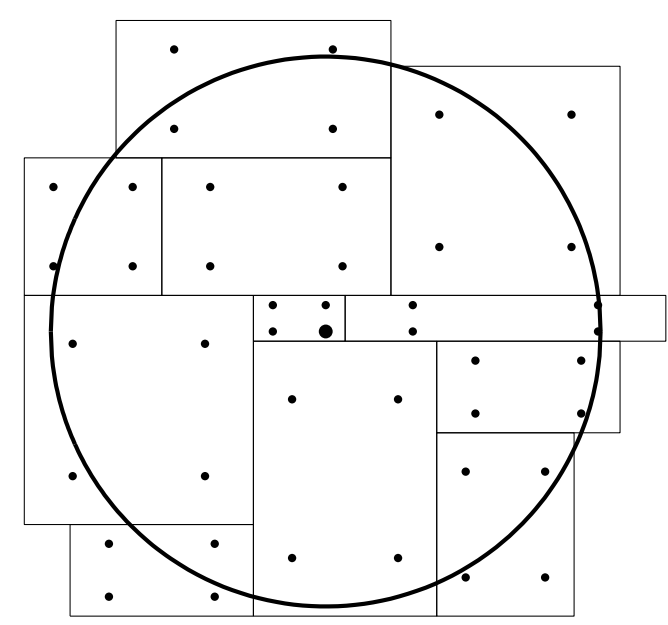

Figure 3: Covering of the peridynamic horizon by rectangles and corresponding Gauß points

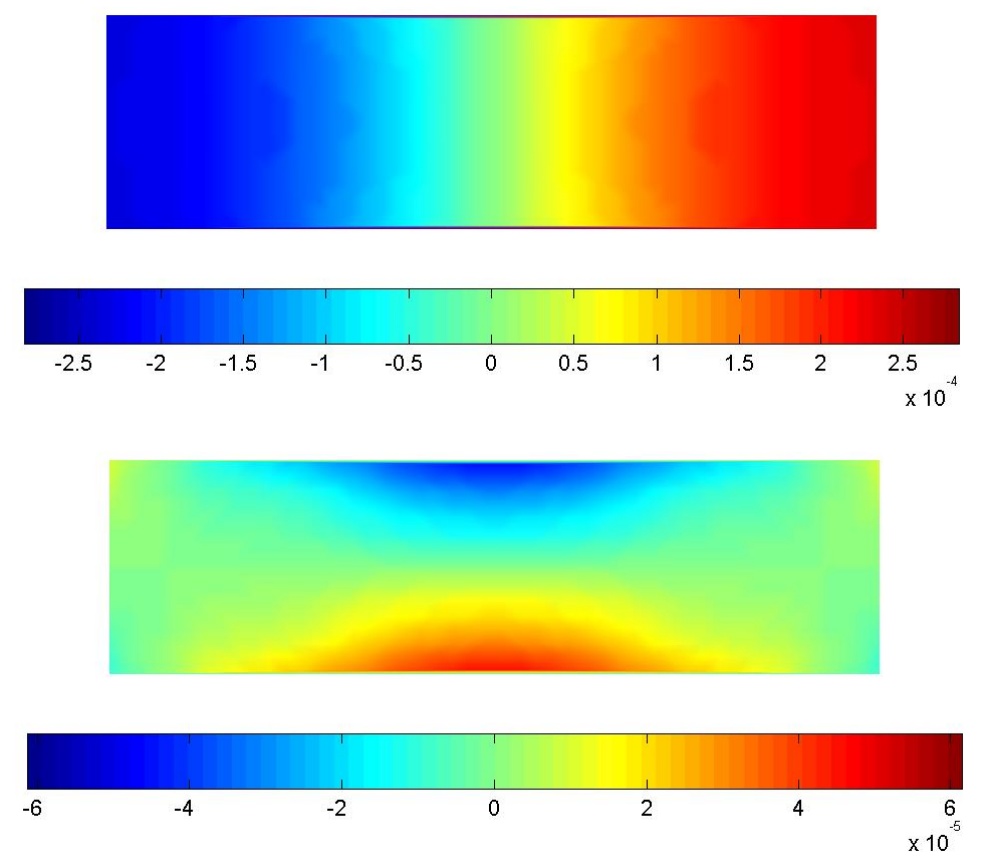

Figure 4: Displacement in $x$ - (above) and $y$-direction (below) at time $t=0.001 \mathrm{~s}$

In the following, we show two simulations done with Silling's Emu code (cf. [41]) that is based upon the composite midpoint rule and employs the model of the prototype microelastic brittle material.

The first simulation shows a rubber plate $\left(\rho \equiv 1000 \mathrm{kgm}^{-3}, E=3 \cdot 10^{6} \mathrm{~Pa}, \nu=1 / 4\right)$ with a slit under constant tension. We have used $50 \times 50 \times 5$ quadrature points.

The next simulation shows the evolution of a defect in a steel bar $\left(\rho \equiv 8000 \mathrm{kgm}^{-3}, E=\right.$ $2.43 \cdot 10^{11} \mathrm{~Pa}, \nu=1 / 4$ ) under tension at the front edge. For the simulation, $30 \times 60 \times 30$, quadrature points have been used. 

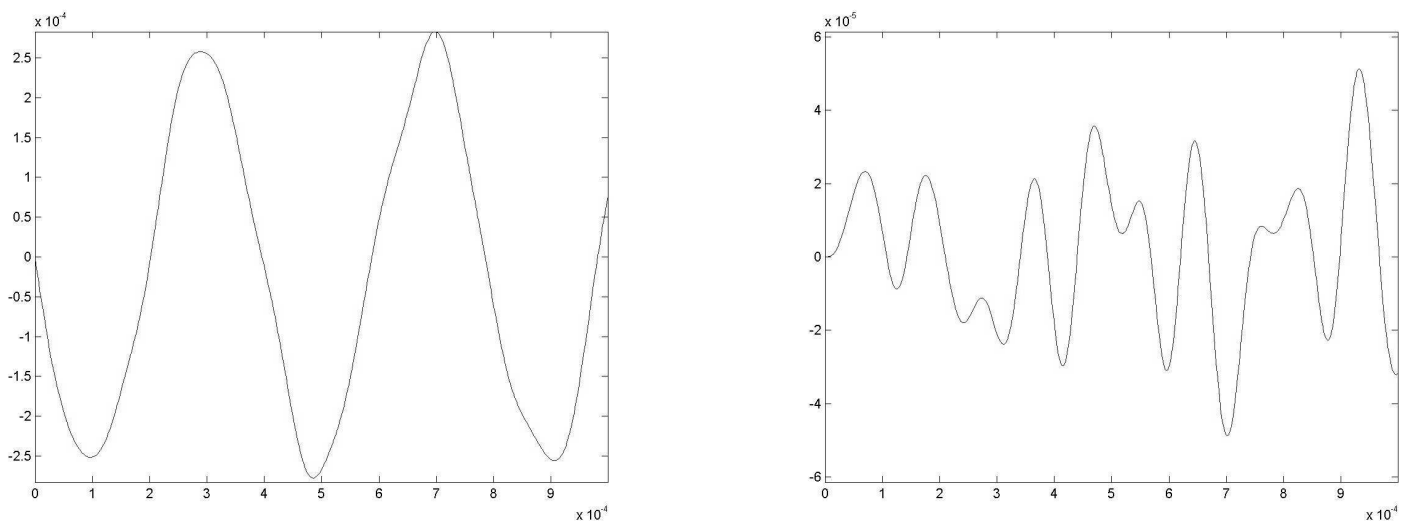

Figure 5: $x$ - (left) and $y$-component (right) of the movement of the first Gauß point in the lower left corner over time
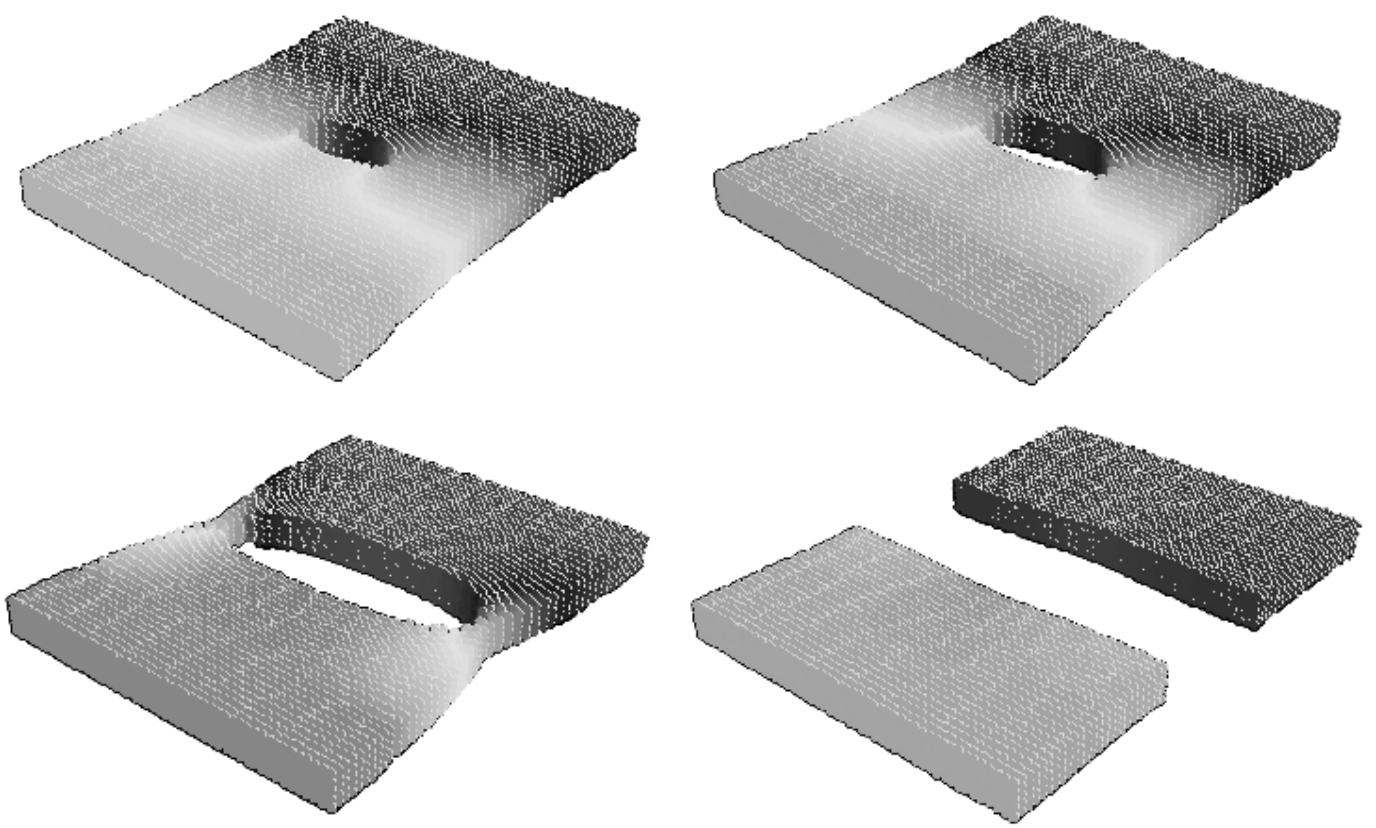

Figure 6: Displacement in $y$-direction at different times

\section{ACKNOWLEDGEMENT}

The authors are indebted to Stephan Kusche and Henrik Büsing for helpful discussions and carrying out parts of the numerical simulations.

\section{REFERENCES}

[1] B. S. Altan, Uniqueness of initial-boundary value problems in nonlocal elasticity. Int. J. Solids Structures 25 (1989) 11, pp. 1271-1278.

[2] B. S. Altan, Uniqueness in nonlocal thermoelasticity. J. Thermal Stresses 14 (1991), pp. 121-128. 

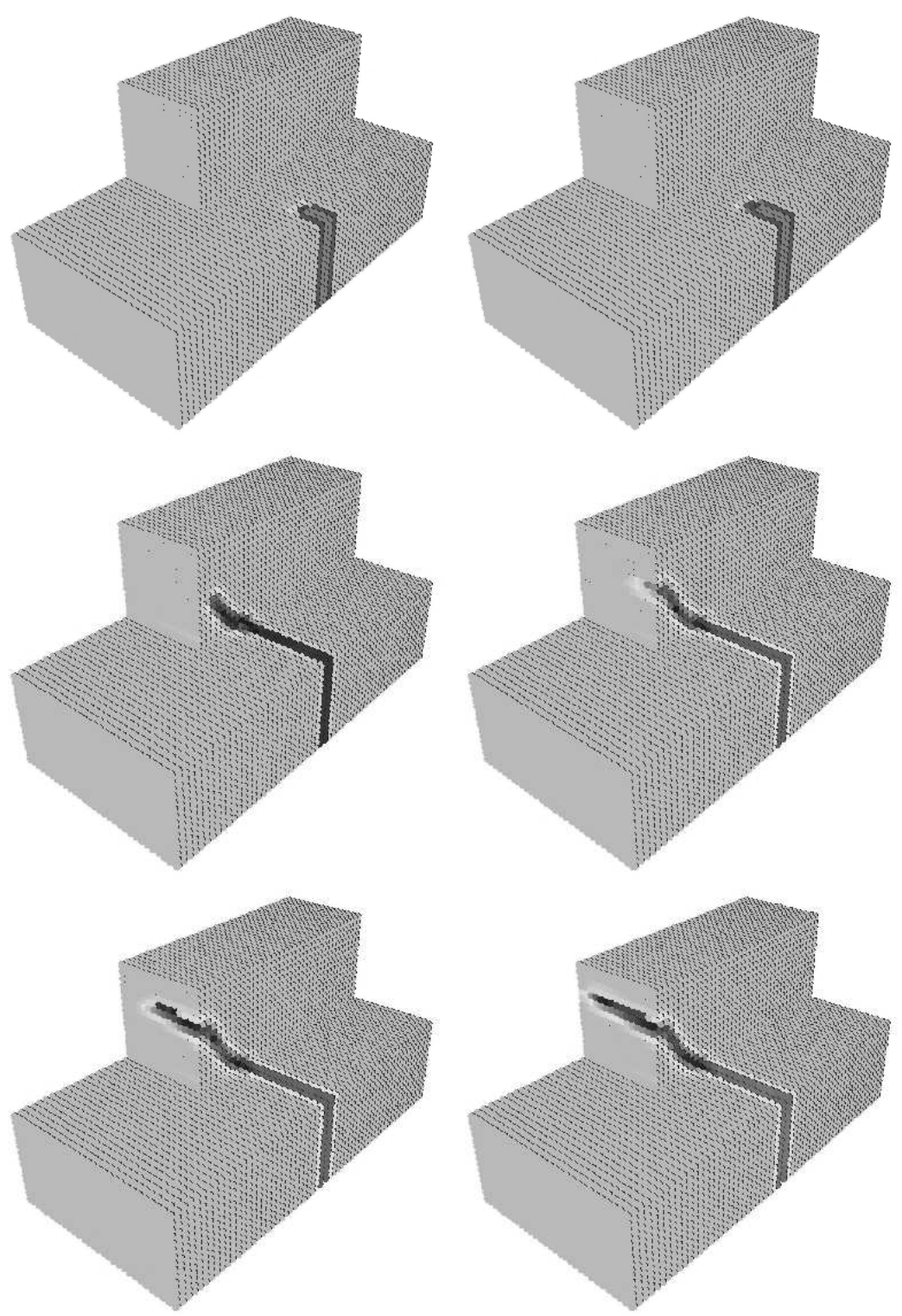

Figure 7: Damage in $y$-direction at different times

[3] H. Amann, Ordinary Differential Equations. de Gruyter, Berlin, 1990.

[4] J. M. Appell, A. S. Kalitvin, and P. P. Zabrejko. Partial Integral Operators and Integrodifferential Equations. M. Dekker, New York, 2000.

[5] M. Arndt and M. Griebel, Derivation of higher order gradient continuum models from atomistic models for crystalline solids. Multiscale Model. Simul. 4 (2005) 2, pp. 531-562.

[6] K. E. Atkinson, The Numerical Solution of Integral Equations of the Second Kind. Cambridge University Press, Cambridge, 1997.

[7] K. Atkinson, A survey of numerical methods for solving nonlinear integral equations. $J$. Int. Eqns.\& Applics. 4 (1992), 15-46. 
[8] Z. P. Bažant and M. Jirásek, Nonlocal integral formulations of plasticity and damage: survey and progress. J. Eng. Mech. 128 (2002) 11, pp. 1119-1149.

[9] T. Belytschko, Y. Y. Lu, and L. Gu, Element-free Galerkin methods. Int. J. Numer. Meth. Engng. 37 (1994), 229-256.

[10] X. Blanc, C. Le Bris, and F. Legoll, Analysis of a prototypical multiscale method coupling atomistic and continuum mechanics. M2AN 39 (2005) 4, pp. 797-826.

[11] F. Bobaru and S. A. Silling, Peridynamic 3D problems of nanofiber networks and carbon nanotube-reinforced composites. Materials and Design: Proc. Numiform 2004, American Institute of Physics, pp. 1565-1570.

[12] F. Bobaru, S. A. Silling, and H. Jiang, Peridynamic fracture and damage modeling of membranes and nanofiber networks. Proc. XI Int. Conf. Fract., Turin, 2005, pp. 5748:1-6.

[13] H. Brunner, Collocation Methods for Volterra Integral and Related Functional Differential Equations Cambridge University Press, Cambridge, 2004.

[14] C. Chen and T. Shih, Finite Element Methods for Integrodifferential Equations. World Scientific, Singapore, 1998.

[15] Y. Chen, J. D. Lee, and A. Eskandarian, Atomistic viewpoint of the applicability of microcontinuum theories. Int. J. Solids Structures 41 (2004) 8, pp. 2085-2097.

[16] Y. Chen, J. D. Lee, and A. Eskandarian, Dynamic meshless method applied to nonlocal crack problems. Theor. Appl. Fracture Mech. 38 (2002), pp. 293-300.

[17] K. Dayal and K. Bhattacharya, Kinetics of phase transformations in the peridynamic formulation of continuum mechanics. Submitted to J. Mech. Phys. Solids, November 2005.

[18] E. Emmrich, Gewöhnliche und Operator-Differentialgleichungen: Eine integrierte Einführung in Randwertprobleme und Evolutionsgleichungen für Studierende. Vieweg, Wiesbaden, 2004.

[19] E. Emmrich and O. Weckner, Analysis and numerical approximation of an integrodifferential equation modelling non-local effects in linear elasticity. Math. Mech. Solids (2005), published online first, DOI: 10.1177/1081286505059748.

[20] A. C. Eringen, Vistas of nonlocal continuum physics. Int. J. Eng. Sci. 30 (1992) 10, pp. 1551-1565.

[21] W. Gerstle and N. Sau, Peridynamic modeling of concrete structures. Proc. 5th Int. Conf. Fract. Mech. Concr. Struct., In: L. Li et al. (eds.), Ia-FRAMCOS 2 (2004), pp. 949-956.

[22] W. Gerstle, N. Sau, and S. A. Silling, Peridynamic modeling of plain and reinforced concrete structures. SMiRT18: 18th Int. Conf. Struct. Mech. React. Technol., Beijing, 2005.

[23] K. F. Graff, Wave Motion in Elastic Solids. Dover Publ., New York, 1991.

[24] M. Griebel et al., Numerische Simulation in der Moleküldynamik. Springer, Berlin, 2004. 
[25] M. Griebel and M. A. Schweitzer, Eds., Meshfree Methods for Partial Differential Equations. Lecture Notes in Computational Science and Engineering 26, Springer, Berlin, 2003.

[26] M. Griebel and M. A. Schweitzer, Eds., Meshfree Methods for Partial Differential Equations II. Lecture Notes in Computational Science and Engineering 43, Springer, Berlin, 2005.

[27] E. Hairer, C. Lubich, and G. Wanner. Geometric Numerical Integration: StructurePreserving Algorithms for Ordinary Differential Equations. Springer, Berlin, 2004.

[28] R. Kress, Linear Integral Equations. Springer, New York, 2nd edition 1999.

[29] E. Kröner, Elasticity theory of materials with long range forces. Int. J. Solids Structures 3 (1967), pp. 731-742.

[30] I. A. Kunin, Elastic Media with Microstructure, vol. I and II. Springer, Berlin, 1982/83.

[31] V. Lakshmikantham and M. R. M. Rao, Theory of Integro-differential Equations. Gordon and Breach, Lausanne, 1995.

[32] C. Le Bris and P. L. Lions, From atoms to crystals: a mathematical journey. Bulletin of the American Mathematical Society 42 (2005) 3, pp. 291-363.

[33] Y. Lei, M. I. Friswell, and S. Adhikari, A Galerkin method for distributed systems with non-local damping. Int. J. Solids Structures (2005), published online first, DOI: 10.1016/j.ijsolstr.2005.06.058.

[34] M. Marder, Supersonic rupture of rubber. arXiv:cond-mat/0504613 v1, April 2005.

[35] A. A. Pisano and P. Fuschi, Closed form solution for a nonlocal elastic bar in tension. Int. J. Solids Structures 40 (2003) 1, pp. 13-23.

[36] C. Polizzotto, Nonlocal elasticity and related variational principles. Int. J. Solids Structures 38 (2001) 42-43, pp. 7359-7380.

[37] T. Rabczuk and T. Belytschko, Cracking particles: a simplified meshfree method for arbitrary evolving cracks. Int. J. Numer. Meth. Engng. 61 (2004), 2316-2343.

[38] D. Rogula, Nonlocal Theory of Material Media. Springer, Berlin, 1982.

[39] S. A. Silling, Reformulation of elasticity theory for discontinuities and long-range forces. J. Mech. Phys. Solids 48 (2000) 1, pp. 175-209.

[40] S. A. Silling, Dynamic fracture modeling with a meshfree peridynamic code. In: K. J. Bathe (ed.), Computational Fluid and Solid Mechanics, Elsevier, Amsterdam, 2003, pp. 641-644.

[41] S. A. Silling, About Emu. http://www. sandia.gov/emu/emu.htm.

[42] S. A. Silling and E. Askari, Peridynamic modeling of impact damage. In: F. J. Moody (ed.), PVP-Vol. 489, American Society of Mechanical Engineers, New York, 2004, pp. 197-205. 
[43] S. A. Silling and E. Askari, A meshfree method based on the peridynamic model of solid mechanics. Computers \& Structures 83 (2005) 17-18, pp. 1526-1535.

[44] S. A. Silling and F. Bobaru, Peridynamic modeling of membranes and fibers. Int. J. Nonlinear Mech. 40 (2005) 2-3, pp. 395-409.

[45] R. D. Skeel, G. Zhang, and T. Schlick. A family of symplectic integrators: stability, accuracy, and molecular dynamics applications. SIAM J. Sci. Comput. 18 (1997) 1, pp. $203-$ 222.

[46] A. M. Stuart and A. R. Humphries. Dynamical Systems and Numerical Analysis. Cambridge University Press, Cambridge, 1998.

[47] S. A. Silling, M. Zimmermann, and R. Abeyaratne, Deformation of a peridynamic bar. J. Elasticity 73 (2003), pp. 173-190.

[48] J. Wang and R. S. Dhaliwal, Uniqueness in generalized nonlocal thermoelasticity. J. Thermal Stresses 16 (1993), pp. 71-77.

[49] J. Wang and R. S. Dhaliwal, On some theorems in the nonlocal theory of micropolar elasticity. Int. J. Solids Structures 30 (1993) 10, pp. 1331-1338.

[50] O. Weckner and R. Abeyaratne, The effect of long-range forces on the dynamics of a bar. J. Mech. Phys. Solids 53 (2005) 3, pp. 705-728.

[51] O. Weckner and E. Emmrich, Numerical simulation of the dynamics of a nonlocal, inhomogeneous, infinite bar. J. Comp. Appl. Mech. 6 (2005) 2, pp. 311-319.

[52] O. Weckner and E. Emmrich, Energy conserving spatial discretisation methods for the peridynamic equation of motion in non-local elasticity theory. Preprint 25-2005, Institute of Mathematics, TU Berlin, September 2005.

[53] M. Zimmermann, A continuum theory with long-range forces for solids. PhD Thesis, Massachusetts Institute of Technology, Department of Mechanical Engineering, 2005. 